\title{
High-grade pT2 disease
}

Cite as: Can Urol Assoc J 2013;7(11-12):e779. http://dx.doi.org/10.5489/cuaj.1791

In the CUA Guideline on postoperative surveillance for upper tract urothelial carcinoma, ${ }^{1}$ there was an omission in Table 1.

In the recommended protocol for follow-up after surgery for UTUC, the second row should have been: HG $\mathrm{pT}<2$ $\mathrm{Nx} / 0$ or LG/HG pT2 Nx/0 (Table 1). This now accounts for patients with high-grade pT2 disease.

\section{Reference}

1. Kapoor $A$, Allard $C B$, Black $P$, et al. Canadian guidelines for postoperative surveillance of upper urinary tract urothelial carcinoma. Can Urol Assoc J 2013;7:306-11. http://dx.doi.org/10.5489/cuaj.1578

\begin{tabular}{|c|c|c|c|c|c|c|c|c|c|c|}
\hline \multirow[t]{2}{*}{ Pathology } & \multirow[t]{2}{*}{ Investigations } & \multicolumn{9}{|c|}{ No. months after surgery for UTUC } \\
\hline & & 3 & 6 & 12 & 18 & 24 & 30 & 36 & 48 & 60 \\
\hline \multicolumn{11}{|c|}{ LG pT<2 Nx/0 } \\
\hline & $\mathrm{Hx}$ and $\mathrm{PE}$ & $x$ & $x$ & $\mathrm{x}$ & $x$ & $x$ & & $x$ & $\mathrm{x}$ & $\mathrm{x}$ \\
\hline & Blood work & $x$ & $x$ & $x$ & $x$ & $x$ & & $\mathrm{x}$ & $\mathrm{x}$ & $x$ \\
\hline & Urine cytology & $x$ & $x$ & $x$ & $x$ & $x$ & & $x$ & $x$ & $x$ \\
\hline & Cystoscopy & $x$ & $x$ & $x$ & $x$ & $x$ & & $x$ & $x$ & $x$ \\
\hline & CXR & & & $x$ & & $x$ & & $x$ & $\mathrm{x}$ & $x$ \\
\hline & CTU & & & $x$ & & $x$ & & $\mathrm{x}$ & $\mathrm{x}$ & $x$ \\
\hline & \pm Ureteroscopy* & $x$ & $x$ & $x$ & $\mathrm{x}$ & $x$ & & $x$ & $\mathrm{x}$ & $x$ \\
\hline \multicolumn{11}{|c|}{$\begin{array}{l}\text { HG pT<2 Nx/0 or } \\
\text { LG/HG pT2 Nx/0 }\end{array}$} \\
\hline & $\mathrm{Hx}$ and $\mathrm{PE}$ & $x$ & $x$ & $x$ & $x$ & $x$ & & $x$ & $x$ & $\mathrm{x}$ \\
\hline & Blood work & $x$ & $x$ & $x$ & $x$ & $x$ & & $x$ & $x$ & $x$ \\
\hline & Urine cytology & $x$ & $x$ & $x$ & $x$ & $x$ & & $x$ & $\mathrm{x}$ & $x$ \\
\hline & Cystoscopy & $x$ & $x$ & $x$ & $x$ & $x$ & & $x$ & $x$ & $x$ \\
\hline & CXR & & $x$ & $x$ & $x$ & $x$ & & $\mathrm{x}$ & $x$ & $x$ \\
\hline & CTU & & $x$ & $x$ & $x$ & $x$ & & $x$ & $x$ & $x$ \\
\hline & \pm Ureteroscopy* & $x$ & $x$ & $x$ & $\mathrm{x}$ & $x$ & & $x$ & $x$ & $x$ \\
\hline \multicolumn{11}{|c|}{ LG/HG pT>2 or pN+ } \\
\hline & $\mathrm{Hx}$ and $\mathrm{PE}$ & $x$ & $x$ & $x$ & $x$ & $x$ & & $\mathrm{x}$ & $x$ & $x$ \\
\hline & Blood work & $x$ & $x$ & $x$ & $x$ & $x$ & & $x$ & $x$ & $x$ \\
\hline & Urine cytology & $x$ & $x$ & $x$ & $x$ & $x$ & & $x$ & $x$ & $x$ \\
\hline & Cystoscopy & $x$ & $x$ & $x$ & $x$ & $x$ & & $x$ & $x$ & $x$ \\
\hline & CXR & $x$ & $x$ & $x$ & $x$ & $x$ & $x$ & $x$ & $x$ & $x$ \\
\hline & CTU & $x$ & $x$ & $x$ & $x$ & $x$ & $x$ & $x$ & $x$ & $x$ \\
\hline & \pm Ureteroscopy* & $x$ & $x$ & $x$ & $\mathrm{x}$ & $x$ & & $x$ & $x$ & $x$ \\
\hline
\end{tabular}

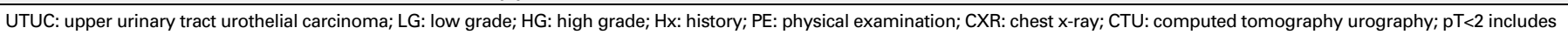
pTis, pTa and pT1; *Ipsilateral ureteroscopy with selective cytology or biopsy should be performed following nephron-sparing procedures. 